\title{
Evolutionary optimization of network reconstruction from derivative-variable correlations
}

\author{
Marc G. Leguia ${ }^{1,2}$, Ralph G. Andrzejak ${ }^{2,3}$, Zoran Levnajić ${ }^{1,4}$ \\ Faculty of Information Studies in Novo mesto, Novo Mesto, Slovenia (1) \\ Department of Information and Communication Technologies, University Pompeu \\ Fabra, Barcelona, Spain (2) \\ Institute for Bioengineering of Catalonia (IBEC), Barcelona, Catalonia, Spain (3) \\ Department of Knowledge Technologies, Jozef Stefan Institute, Slovenia (4)
}

\begin{abstract}
Topologies of real-world complex networks are rarely accessible, but can often be reconstructed from experimentally obtained time series via suitable network reconstruction methods. Extending our earlier work on methods based on statistics of derivative-variable correlations, we here present a new method built on integrating an evolutionary optimization algorithm into the derivative-variable correlation method. Results obtained from our modification of the method in general outperform the original results, demonstrating the suitability of evolutionary optimization logic in network reconstruction problems. We show the method's usefulness in realistic scenarios where the reconstruction precision can be limited by the nature of the time series. We also discuss important limitations coming from various dynamical regimes that time series can belong to.
\end{abstract}

\section{Introduction}

Network science has been a rapidly growing field over the last decades, providing valuable contributions in natural and social sciences $[1,2]$. Its core paradigm is to represent the entities (units) of some complex system as nodes and model the interactions among these units as network links. With examples ranging from social or economic networks to networks of neurons or proteins, this field involves a new methodological framework that includes dynamical systems and statistical physics on one side, and graph theory and algorithms on the other side.

In general, the functioning of a natural, social or technological network can be viewed as a cumulative effect of its structure (topology of connections among nodes/units) and dynamics (interactions/relationships among these nodes). For example, the operation of gene regulation network is an intricate combination of structure (which genes influence one another) and dynamics (details of gene activation and repression). Therefore, in order to fully understand the functioning of a complex network, one needs information on both its structure and its dynamics.

However, our knowledge about the structure of real networks is often unreliable, mainly due to incomplete accessibility, which leads to missing or spurious links [3, 4]. 
On the other hand, data on dynamics can often be measured with better precision, but inferring the structure from dynamical data is far from simple. This calls for the development of methods for reconstructing network structure from the available dynamical data. Knowledge about the network structure is not just useful for understanding its functioning, but can help designing methods for its control or longterm prediction of its behavior. Also, grasping architecture principles of real networks, especially those that underwent evolution (e.g. biological systems) gives inspiration for engineering artificial networks with prescribed functions.

And indeed, a lot of research has been done aiming to infer the topology of complex systems from time series that capture the network dynamics [5-9]. Some are focused on Kuramoto-like systems [10], where the system can synchronize [11]. In that case the reconstruction is not trivial because the synchronization overshadows most of the information of the system. These methods hence often employ driving or phase resets to infer the topology [12-14]. Contrary to the methods developed for the study of networks of phase oscillators, there is a range of methods applicable to general dynamical systems $[6,15,16]$. The study of reconstructing networks that varies in time also has been a subject of interest [17]. Some reconstruction methods are based on power expansions [18] or Laplacian spectra [19]. Several methods are invasive, i.e., rely on perturbing the system $[12,13]$, which in practice is often difficult or even impossible. Moreover, some methods make use of large dynamical data sets, which is rarely experimentally feasible. Indeed, this field calls for more research, particularly by exploring other known methods from other fields that could be of use for the reconstruction problems. Along those lines is the non-invasive method proposed in [15], which is applicable for short data sets.

In this paper we present the extension of a derivative-variable correlations method described in [15]. Our approach tries to improve the results in [15] by exploiting the simulated annealing (evolutionary optimization algorithm). Our goal is also to emphasize the benefits of employing evolutionary algorithms for reconstruction problems. In fact, the logic of simulated annealing fits naturally into the network reconstruction problem, since the mutations of the reconstructed adjacency matrix evolve according to the fit between the real and reconstructed trajectories.

\section{The derivative-variable correlation method}

Here we briefly summarize the derivative-variable correlation method [15]. We consider a general complex dynamical system composed of $N$ nodes, whose dynamical states are represented by the variables $x_{i}$. We assume that the network dynamics can be described by:

$$
\dot{x}_{i}=f_{i}\left(x_{i}\right)+\sum_{j=1}^{N} A_{j i} h_{j}\left(x_{j}\right),
$$

where $\dot{x}_{i}$ is the time-derivative of $x_{i}$, the function $f_{i}$ represents the local dynamics, and $h_{j}$ is the inter-node interaction, which expresses how node $j$ acts on the other nodes. 
The adjacency matrix $A_{i j}$ represents the structure of the network by capturing the connections between node $i$ and $j$. It is this adjacency matrix that we seek to find. We assume that:

(i) The system behaves as Eq.(1),

(ii) The functions $f_{i}$ and $h_{j}$ are known,

After setting these assumptions, we define the following correlation matrices:

$$
\begin{aligned}
& B=<g\left(x_{i}\right) \dot{x_{j}}>, \\
& C=<g\left(x_{i}\right) f_{j}\left(x_{j}\right)>, \\
& E=<g\left(x_{i}\right) h_{j}\left(x_{j}\right)>.
\end{aligned}
$$

Where $<\cdot>$ represents time-averaging, the indices goes from $i, j=(1,2 \ldots N)$ and $g(x)$ is a function that plays a key role in maximizing the precision of the reconstruction. Its explicit details will be explained later. If we multiply Eq.(1) by $g$ we get:

$$
g\left(x_{k}\right) \dot{x}_{i}=g\left(x_{k}\right) f_{i}\left(x_{i}\right)+\sum_{j=1}^{N} A_{j i} g\left(x_{k}\right) x_{j} .
$$

If we make averages across time, we can identify the matrices defined in Eq.(2) and rewrite Eq.(3) as:

$$
R=E^{-1}(B-C)
$$

where $R$ represents the reconstructed adjacency matrix. This equation is always applicable as long as the system behaves as Eq.(1). The matrices we defined in Eq.(2) can always be computed since our observable are $L$ values $\left(x_{i}\left(t_{1}\right) \ldots x_{i}\left(t_{L}\right)\right)$ for each of the $N$ nodes. These measurements are equally separated in time by $\tau$. For infinitely long time series, the choice of $g$ become less important. In realistic scenarios, however, where the time series are short and/or noisy, the selection of $g$ plays the crucial role by allowing for tuning of the reconstruction equation above. In [15] the function $g$ was taken to be a periodic function on the interval covered by our observable data. For simplicity, $g$ was defined as the first 10 Fourier components:

$$
g(x)=\sum_{m=1}^{10}\left[a_{m} \sin (m x)+b_{m} \cos (m x)\right] .
$$

Tuning of the reconstruction Eq.(4) was implemented by choosing the coefficients $a_{m}$ and $b_{m}$ at random (many times) and then use the $g$ 's which lead to best precision. To quantify the precision for various realizations of $g$, we define the reconstruction error $\Delta_{A}$ as the deviation of the reconstructed adjacency matrix $R$ from the original adjacency matrix $A$ :

$$
\Delta_{A}=\sqrt{\frac{\sum_{i j}\left(R_{i j}-A_{i j}\right)^{2}}{\sum_{i j} A_{i j}^{2}}} .
$$

In real-world applications the matrix $A$ is of course unknown, so accordingly, $\Delta_{A}$ cannot be calculated directly. We need another way to quantify the precision of our 
measurements. To do so, we use $R$ to check how well we reproduce our original data. For instance we start the dynamics from $x_{i}\left(t_{1}\right)$ and run Eq.(1) using $R$ as an estimate for $A$ until $t=t_{2}$ to obtain $y_{i}\left(t_{2}\right)$. Then we restart the run with $x_{i}\left(t_{2}\right)$ until $t=t_{3}$ obtaining $y_{i}\left(t_{3}\right)$ and so on. The discrepancy between the reconstructed time series $y_{i}\left(t_{l}\right)$ and the original time series $x_{i}\left(t_{l}\right)$ is a measure of the reconstruction precision based only on the available data. This leads to the definition of the trajectory error $\Delta_{T}$ as:

$$
\Delta_{T}=\frac{1}{N} \sum_{i=1}^{N} \sqrt{\frac{<\left(x_{i}-y_{i}\right)^{2}>}{<\left(x_{i}-<x_{i}>\right)^{2}>}} .
$$

Where $<\cdot>$ represents time-averaging. One of the key results from [15] is that in general, $\Delta_{A}$ and $\Delta_{T}$ are correlated. Thus, low values of $\Delta_{T}$ generally imply low values of $\Delta_{A}$. Each different choice of the coefficients $a_{m}$ and $b_{m}$ in Eq.(5) leads to a different reconstruction precision. In other terms, $g$ is a tunable parameter that we can use to maximize the precision, by considering only the choices of $g$ that lead to minimal $\Delta_{T}$. In contrast to [15], we will here not be choosing the Fourier coefficients at random, but will instead apply a simulated annealing algorithm to mutate the Fourier coefficients. As we show in what follows, this indeed in general leads to an enhanced performance.

\section{Generalization of the method via simulated annealing}

It is easy to acknowledge that taking a big sample of random functions $g$ and using each of them with Eq.(4) might not be the optimal approach. The search space we are dealing with is not known at all and is in general very rugged. So even a very large number of random guesses for $g$, does not guarantee a sufficient exploration of the search space.

Fortunately, scenarios like this - because of their notoriety - are well studied, particularly in computer science, with a range of heuristics applicable depending on the specific case at hand. Among the most standard ones are evolutionary optimization algorithms, such as simulated annealing [20]. Simulated annealing is an optimization technique that is used to explore rugged and large search spaces while avoiding the local shallow minima [21], used in a vast variety of scientific problems. It is related to the Metropolis-Hastings algorithm [20] often used for statistical mechanics problems. The approach was proven useful also in the network reconstruction scenarios, where its employment leads to reconstruct the essential features of pore geometry and connectivity of chalk [22].

Simulated annealing involves a function (energy) that needs to be minimized and proceeds by systematically modifying (mutating) the proposed solution. Mutations leading to a decrease of energy are accepted and those leading to its increase are either rejected or accepted. One defines a control parameter (temperature) which specifies (typically in a probabilistic way) which worsening mutations are accepted. Accepting none of them can quickly make the system get stuck in a local minimum, while accepting too many of them leads to excessive wandering without ever settling into any minimum. 
How much worsening mutations is best to accept strongly depends on the problem in question, with several different strategies to choose from. In general, one wants to start with a high temperature to allow for a big region of the search space to be initially explored, and then gradually decrease the temperature so that the solution would eventually settle into a minimum. This is in fact the strategy we will use.

Before starting the annealing, we need a proper definition of the cost function (energy) that is to be minimized. In our case this is the trajectory error $\Delta_{T}$, since it is the quantity that can be measured for any reconstructed matrix $R_{i j}$. Hence, the annealing procedure will minimize $\Delta_{T}$, which we expect to be in general correlated with minimization of the adjacency matrix error $\Delta_{A}$. We start the annealing by setting randomly each Fourier coefficient for the function $g$ from a log-uniform probability between $[0,100]$. Then we carry out the following steps:

(i) We start by picking randomly one Fourier coefficient as defined in Eq.(5) (for example, one of $\left.a_{m}-\mathrm{s}\right)$, and modify it as follows: $a_{m}=a_{m}+\alpha a_{m}$, where $\alpha=U / \log (k)$ with $k$ being the current step of the annealing and $U$ random numbers taken from a uniform distribution between [-1:1].

(ii) Compute the modified trajectory error $\Delta_{T}^{\prime}$ using the mutated coefficient $a_{m}$ and compare it to the old trajectory error $\Delta_{T}$, obtaining the difference $\Delta H=\Delta_{T}^{\prime}-\Delta_{T}$,

(iii) Accept the mutation (keep $a_{m}$ ) according to the probability $p$ defined as $[20]$ :

$$
p= \begin{cases}1 & \text { if } \Delta H \leq 0 ; \\ e^{-\Delta H / \beta} & \text { if } \Delta H>0 ;\end{cases}
$$

where $\beta=\beta(k)$ is the temperature, which decreases with annealing steps as specified below,

(iv) Continue the process at point (i) by randomly mutating another Fourier coefficient in the function $g$.

Next we aim to establish the most suitable annealing scheme for our reconstruction problem. To that end, we study two annealing configurations as follows.

(a) The logarithmic temperature decrease

$$
\beta_{\log }=\frac{\beta_{0}}{\ln (k)}
$$

with the initial temperature $\beta_{0}=10^{-5}[23]$.

(b) The exponential temperature decrease

$$
\beta_{\exp }=\beta_{0} \lambda^{k / 10}
$$

with $\lambda=0.99$ and initial temperature $\beta_{0}=10^{-3}$.

In general, scheme (a) gives a very slow temperature decrease which protects against freezing too quickly, but it requires a lot of steps if we want to cover a wider range of temperature. In contrast, scheme (b) gives a much faster convergence to the minimum, but it might miss a better minimum due to fast freezing. In the next section we test both schemes, but start from a lower temperature for logarithmic decreasing so that both schemes would eventually freeze after a comparable number of steps. 
Evolutionary optimization of network reconstruction from derivative-variable correlations6

\section{Results}

To test the performance of our new method and compare it with the old one, we employ a simple toy-model dynamical system containing $N=6$ nodes with Hansel-Sompolinsky dynamics running on it [24]:

$$
\dot{x}_{i}=-x_{i}+\sum_{j=1}^{N} A_{j i} \tanh \left(x_{j}\right) .
$$

The adjacency matrix $A_{i j}$ that we wish to reconstruct is weighted, with weights randomly chosen between -10 and 10 . Starting from random initial conditions we integrate the system between $t=0$ to $t=4$ and extract 20 values of $x_{i}$ for each node $i$, equally spaced in time by $\tau=0.2$. The obtained time series for all nodes are shown in Fig.(3), top left panel.

\subsection{Comparison of two reconstruction schemes}

We start by examining the actual difference between the two reconstructing schemes and between the two annealing schemes. To that end we consider the problem formulated above with time series from Fig.(3) top left panel and run three reconstruction procedures: (i) the random guessing one (from [15]), (ii) simulated annealing with logarithmic temperature decrease, and (iii) the simulated annealing with exponential temperature decrease. We record the decrease of the trajectory error $\Delta_{T}$ for each reconstruction scheme, and plot it in Fig.(1) left panel as function of the number of steps (iterations of the reconstruction scheme). For the case of the random guessing, we are always taking the overall minimum found so far. We have applied an adaptive stopping criteria. This means, that the annealing do not stop at a given number of steps but when the annealing is "frozen" i.e. when the annealing stops accepting any mutation. In our case when during 2000 steps there is no change or the average change is less than an arbitrary but small threshold. This leads the annealing to stop at different number of steps. In the right panel in Fig.(1) we show the histogram of the final $\Delta_{T}$ values for the three reconstruction schemes, obtained from several runs of each scheme. It is clear from both panels of this figure that both annealing schemes on average outperform the random guessing scheme. In fact, the maximum observed $\Delta_{T}$ for $\beta_{\text {exp }}$ is smaller than the minimum $\Delta_{T}$ found for random guessing. As for choosing between the annealing schemes, we from now on rely on $\beta_{\text {exp }}$, since we found systematically better result for that scheme (as illustrated in left panel in Fig.(1)).

To further analyze the benefits of annealing, we compare in Fig.(2) the scatter plot for random guessing against several realizations of annealing scheme. Scatter plots are obtained by identifying each reconstruction step via its $\Delta_{A}$ and $\Delta_{T}$ and representing it with a point in $2 \mathrm{D}$, where the $\mathrm{x}$-coordinate is the trajectory error and the $\mathrm{y}$-coordinate is the adjacency matrix error. We can see how decreasing the trajectory error indeed decreases the adjacency matrix error for all schemes. Practically all realizations of the annealing achieve better results for both $\Delta_{T}$ and $\Delta_{A}$ than the random guessing. This is 

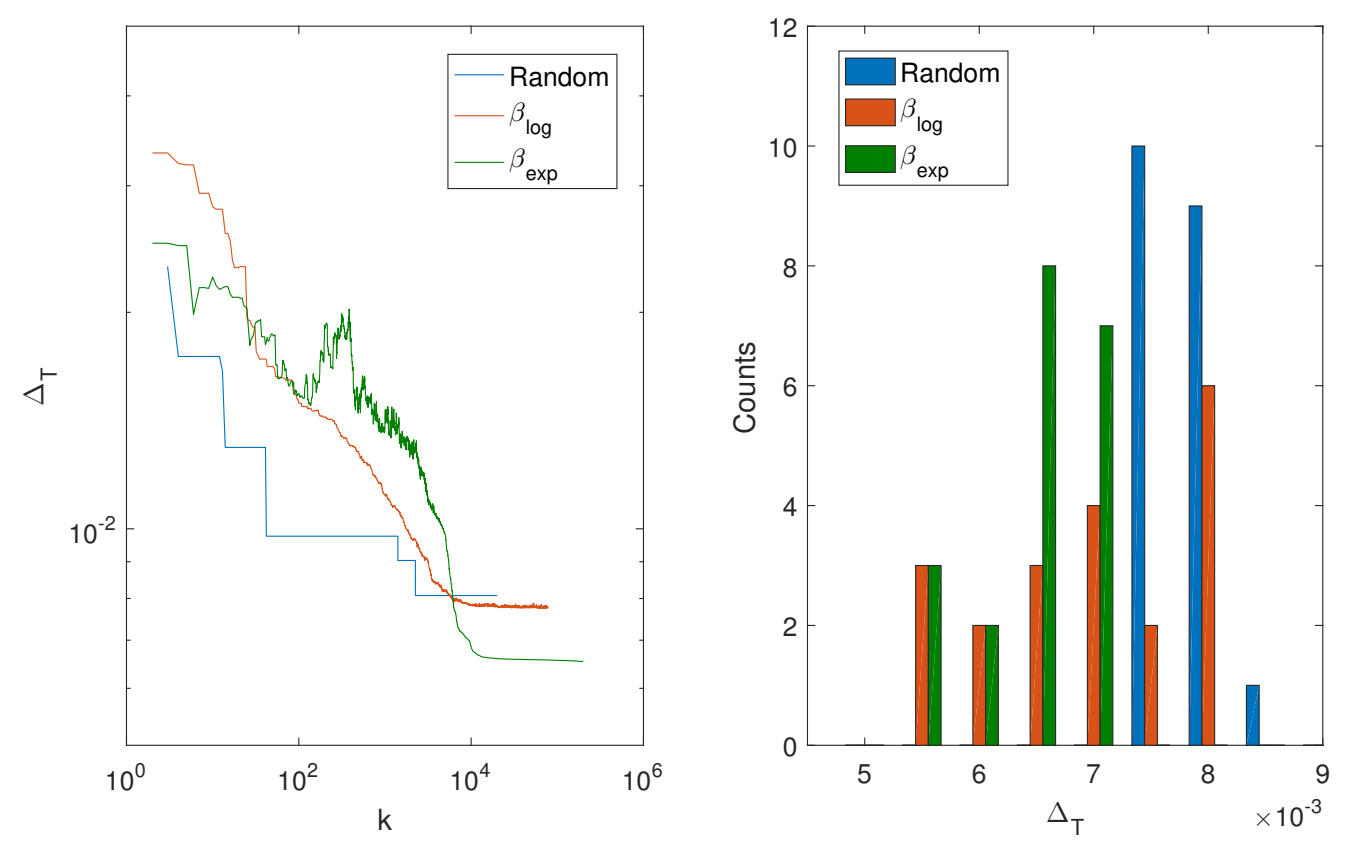

Figure 1. Left: Trajectory error as function of steps for two different annealing schemes $\beta_{\log }$ and $\beta_{\text {exp }}$ and random guessing scheme using one realization of each run. Right: Histogram of the final trajectory errors $\Delta_{T}$ for the same three schemes using 20 independent realizations of each scheme.

further clarified in the inset in $\operatorname{Fig}(2)$. Despite the fact that some realizations converge to similar final precision, we found that each reconstruction comes with its own function $g$. We also found that there is no clear pattern as to which coefficients contribute more to the final precision (This particular result it is not shown in the paper). This nevertheless confirms that, at least in this example, the annealing reconstruction scheme outperforms random guessing scheme.

\subsection{Network reconstruction from different dynamical regimes}

To better appreciate the extent of applicability of random guessing vs. annealing schemes, we next look at how the two reconstruction schemes perform for data from different dynamical regimes. This illustrates the fact that in real experiments the variability of fields [16] of the available data can vary.

To investigate this further, we generate another set of time series from the same system Eq.(8) and show it in top right panel of the Fig.(3). Clearly, these time series do not display as much variability as the one on the top left panel. Two scatter plots obtained using the original random guessing method are shown in the middle two panels of Fig.(3). As expected, the scatter plot in the left gives a far better precision as it reaches to far lower values of both errors $\Delta_{T}$ and $\Delta_{A}$. Moreover, this scatter plot exhibits a more visible correlation between the two errors. This indeed confirms that the left panel time series contain more usable information than the right panel time 
Evolutionary optimization of network reconstruction from derivative-variable correlations 8

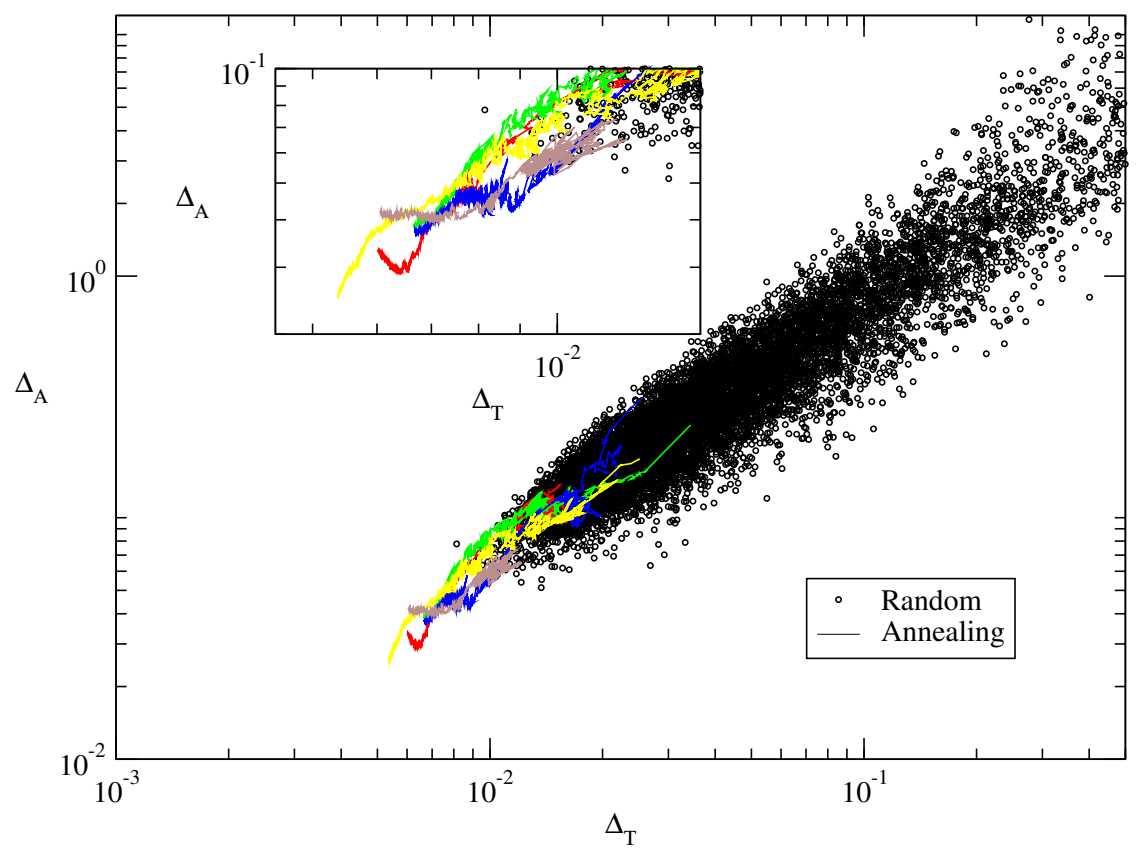

Figure 2. Comparison of the original method using random choices of $g$ (black dots) with several realizations of the simulated annealing approach (colored lines). Minimizing $\Delta_{T}$ in general leads to minimizing $\Delta_{A}$. Inset: Zoom of the final result of the simulated annealing and random guessing.

series, thus allowing for more information to be extracted, which consequently leads to better reconstruction precision.

Next we test the performance of the simulated annealing approach (exponentially temperature decrease) and show the respective evolution plots in the bottom two panels of the Fig.(3) in comparison with the random guessing scatter plots from middle panels. As already observed, in case of time series on the left panel, simulated annealing indeed improves the reconstruction precision compared to random guessing method. All evolutions plots eventually reach smaller final error values. They follow similar paths during the annealing, but ultimately finish in slightly different precision.

In contrast, in case of time series on the right panel, we first observe that the correlation between two errors is far weaker, if present at all. This means that minimizing $\Delta_{T}$ does not in general guarantee minimization of $\Delta_{A}$. And indeed, annealing does not improve the random guessing result. In fact, evolution plots do not follow similar paths and do not reach final points close to each other. Instead, since the quantity to minimize (energy) was the trajectory error $\Delta_{T}$, most evolution plots do improve the trajectory error result, but that does not lead to the improvement of the adjacency matrix error result, due to missing correlation between them. We can conclude that neither the original random guessing method nor the simulated annealing really works with right panel time series. This suggests that complexity (information content) of the time series plays a major role in the reconstruction precision, which merits further research. 
Evolutionary optimization of network reconstruction from derivative-variable correlations9
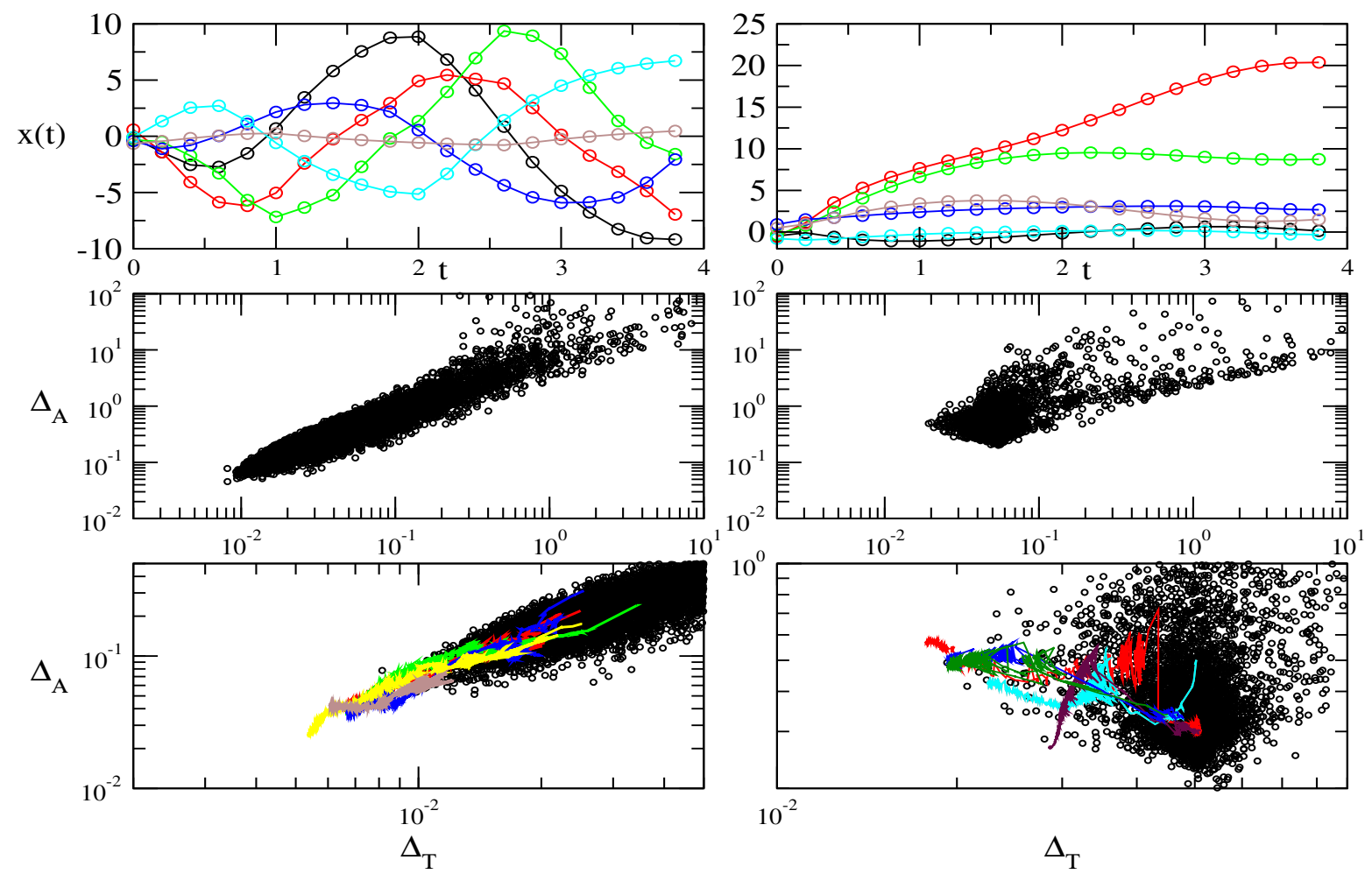

Figure 3. Top panels: two sets of time series generated by the system Eq.(8) (see text). Middle panels: three scatter plots respectively corresponding to three scatter plots obtained via random guessing (old) method. Bottom panels: evolution plots of reconstruction via simulated annealing (several runs in different colors) compared with the scatter plots from middle panels (black dots).

\subsection{Comparison of reconstruction schemes for varying data quality}

At the outset of discussion so far we assumed that the empirical data (time series) come with a given sampling frequency and number of data points (total length of the time series). Yet for a reconstruction method to be of practical use, it should be implementable in realistic scenarios where due to experimental limitations these parameters might not be adjustable. In the remainder of this Section, we investigate the robustness of both reconstruction schemes to these two limitations. For simplicity, we limit ourselves only to the case of time series on the left panel in Fig.(3).

First we examine the impact of the sampling frequency $\tau$. In particular, since measurement frequency is becoming less of a problem for modern experimental equipment, we look at reducing $\tau$ below the original value of $\tau=0.2$. We keep the total number of recorded points fixed to 20. Lowering $\tau$ means that the total recording time no longer goes up to $t=4$, but it is less than that, which means that time series are shorter (in the sense of physical time). This leads to data points covering smaller portion of the dynamical phase space, which is expected to worsen the reconstruction. On the other hand, shrinking $\tau$ makes the derivative estimates better, which is expected to improve the reconstruction. To test this we consider 15 values of $\tau$ between 0 and 0.2 , and run 20 
realizations of each reconstruction scheme for each value of $\tau$. In Fig.(4) we plot the final average values of $\Delta_{T}$ in the left panel and $\Delta_{A}$ in the right panel as a function of $\tau$. The errorbars represent the standard deviation over different realizations. The trajectory

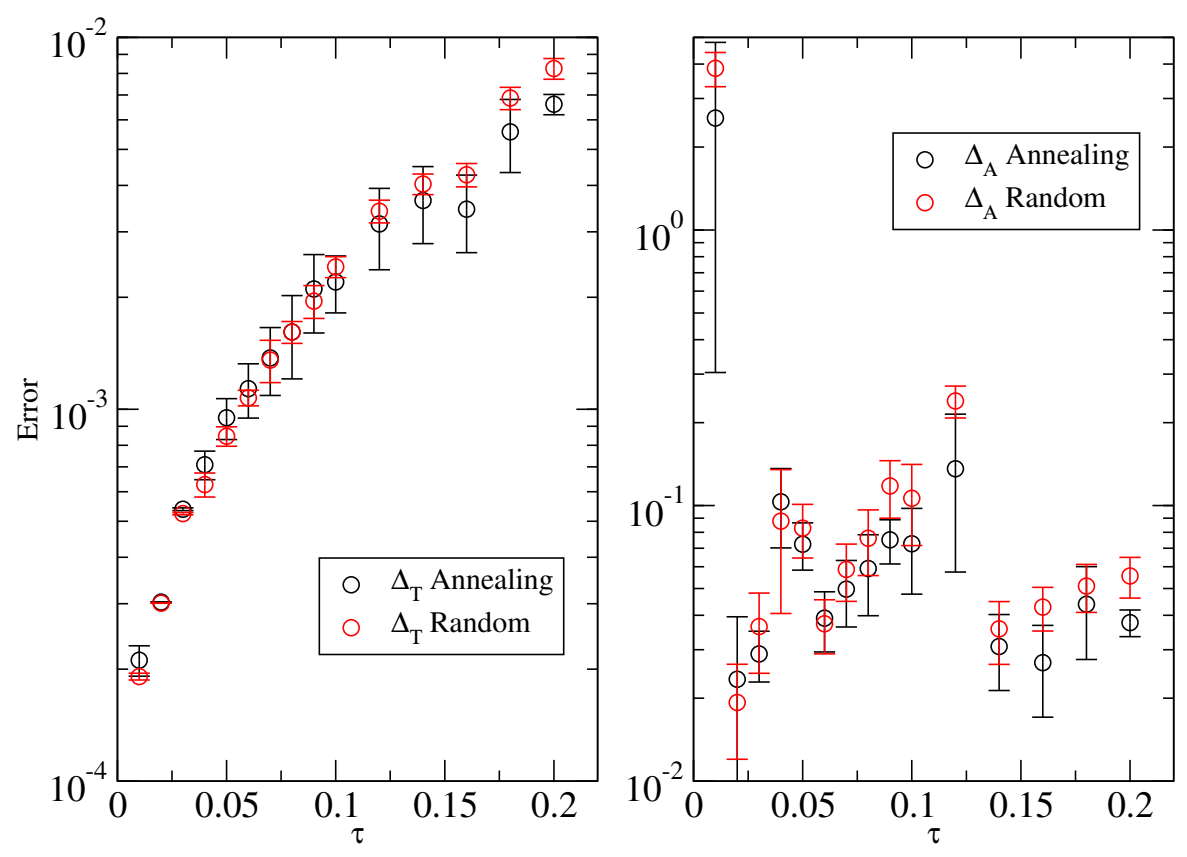

Figure 4. Average obtained value of the trajectory error (left) and adjacency matrix error (right) as a function of the sampling frequency for the random guessing scheme (red) and the annealing scheme (black). We use the time series of the top-left panel in Fig.(3). The errorbars represent the standard deviation of the different realizations obtained over 20 realizations for each scheme and each value of $\tau$.

error displays a well pronounced trend to increase with increasing $\tau$ for both schemes. At larger $\tau$ annealing shows lower trajectory errors. This is largely expected, since adjusting to trajectory is easier with smaller $\tau$, so there annealing makes no significant improvement, and both schemes in fact perform very well. As for the adjacency matrix error in the right panel, there is no clear trend mirroring the pattern found for trajectory error. It seems that actually the precision is worse at smallest considered $\tau=0.01$, then good for $\tau=0.02$ and slightly worsening until $\tau=0.12$, then again good for $\tau=0.14$ and slightly worsening until $\tau=0.2$. This indicates an interplay between phase space coverage (total length of time series) and quality of derivative estimates (resolution), which makes the reconstruction precision change as observed. For instance, it appears that near $\tau=0.12$ exploring a wider region of the phase space gradually becomes more important than the derivative estimates. Interestingly, this interplay is found in both reconstruction schemes, although for increasing $\tau$ annealing seems to gradually perform better than random guessing.

We also studied the performance of reconstruction schemes by varying the total recording time while keeping the recording frequency fixed to $\tau=0.2$. This is to say that the number of data points varies, and consequently the phase space coverage also varies, but the derivative estimates are of unchanged quality. In Fig.(5) we plot the 
trajectory and the adjacency matrix errors as function of the number of points, again using the time series in the top left panel of Fig.(3). Errorbars again represent the standard deviation over 20 different realizations for both schemes. Both $\Delta_{A}$ and $\Delta_{T}$ for both reconstruction schemes improve with the number of points and arrive at a plateau, where adding more points does not improve the precision significantly. This gives an insight into how the method performance reaches the best precision with a certain phase space coverage. This also has to do with intrinsic "reconstructability" of the system. When we do not have enough points (until 12), both the annealing and the random evolution do not have enough information to reconstruct the network and both $\Delta_{A}$ and $\Delta_{T}$ are high. At about 12 data points, the method finally gets enough information to reconstruct the network and precision sharply improves, but then quickly saturates. Again, annealing performs slightly better with increasing number of data points. Note that in this case, the pattern observed for $\Delta_{A}$ and $\Delta_{T}$ is very similar. We conclude by
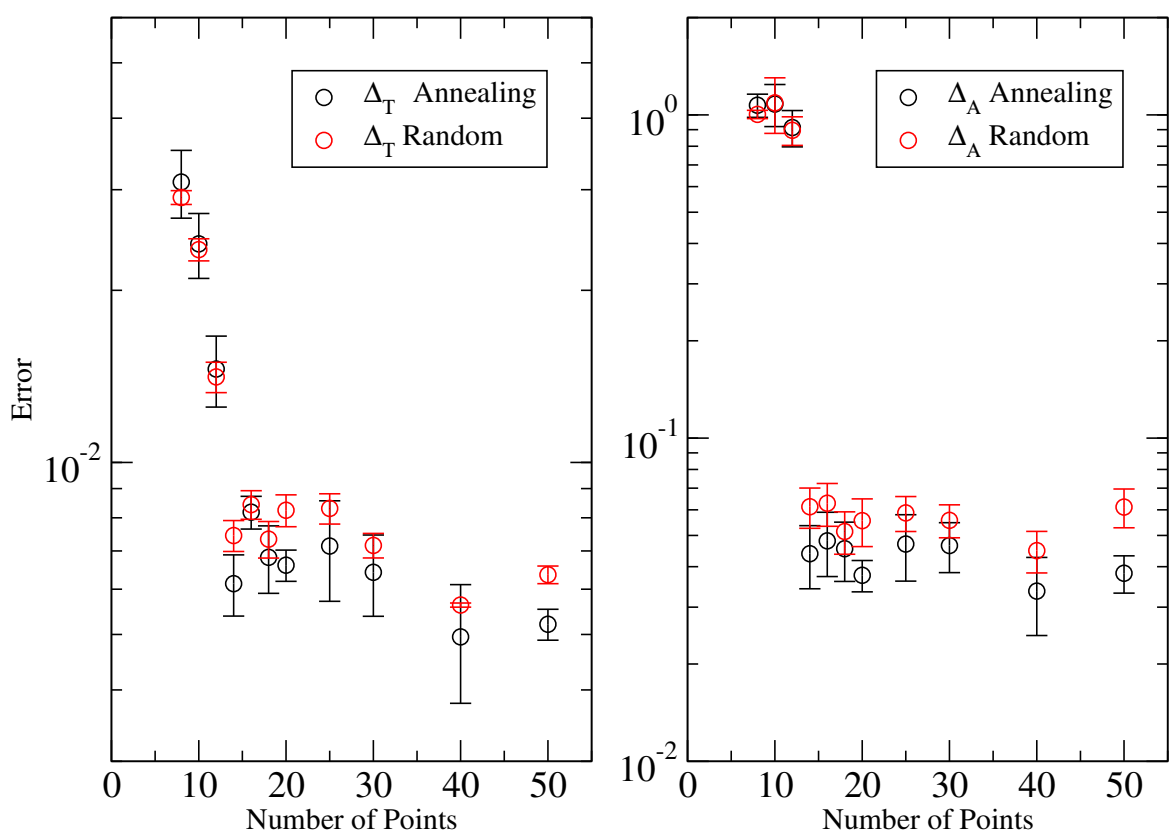

Figure 5. Average obtained value of the trajectory error (left) and adjacency matrix error (right) as a function of the number of points keeping $\tau=0.2$ for he random guessing scheme (red) and the annealing scheme (black). We use the time series of the top left panel in Fig.(3). The errorbars represent the standard deviation of the different realizations obtained over 20 realizations for each scheme and each number of points.

noting that both schemes are in principle applicable in realistic scenarios with scarce data, but annealing scheme - at least with time series here considered - does indeed perform somewhat better. 
Evolutionary optimization of network reconstruction from derivative-variable correlations12

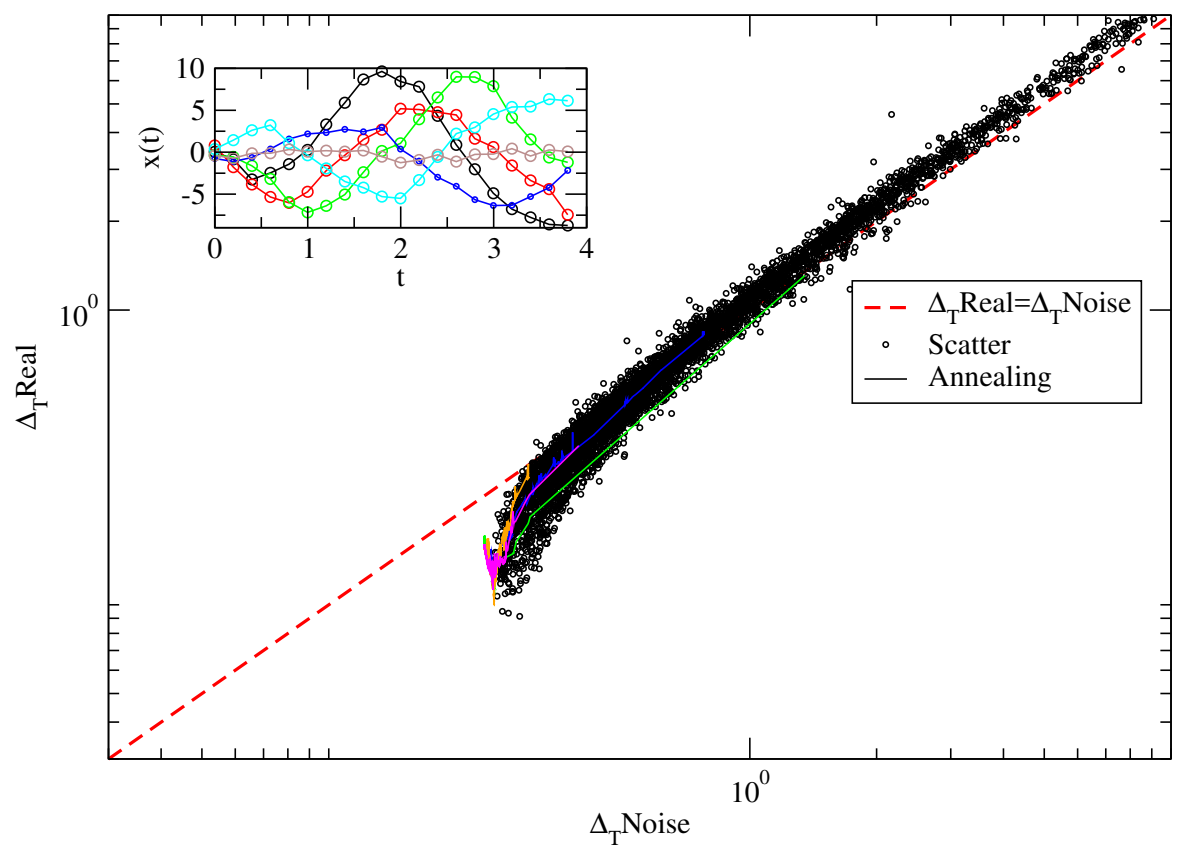

Figure 6. Scatter plot of the error of the noisy trajectories with the error of the real trajectories. The lines correspond to several realizations of the annealing. The dotted line correspond to $\Delta_{T}$ Real $=\Delta_{T}$ Noise relation. Inset: Time series from top left panel in Fig.(3) with the addition of white Gaussian noise with $\sigma^{2}=0.1$.

\subsection{Influence of noise}

Finally we also tested the impact of noise on our method. In [15], the authors applied a smoothing technique to reduce the impact of noise on the method. Here, to complement the approach of [15], we study what happens if we minimize the noisy trajectory error. To do so, we use the time series of the left top panel in Fig.(3) and add white Gaussian noise with $\sigma^{2}=0.1$. These time series are displayed in the inset of Fig.(6). In Fig.(6) we also see the scatter plot of the error of the noisy trajectories with the error of the real trajectories. Using the noisy time series as starting point, the real trajectory error is computed by comparing how the time series generated by the method resembles the noise-free one. When the error is big, there is high correlation (red dotted line). However, once the error of the noisy time series gets smaller, the error of the real trajectories gets smaller at a faster pace. This implies that minimizing directly the error of the noisy trajectories gets you closer to the real trajectories than to the noisy ones. We see this behavior also for the solutions obtained from simulated annealing (lines in Fig.(6)). This can be explained by recalling that the method uses smooth basis functions (Fourier series of order 10) to approximate the noisy signal. Therefore, it cannot approximate well the noisy edges in the curves which are caused by the superposition of the signal with white noise. However, the method still is successful to get the overall shape. And exactly this overall shape is the original signal without noise. Therefore, the trajectory error with regard to the original signal becomes smaller than the one with regard to the noisy time series. We are aware that by using Fourier 
series of higher order, the method could approximate noisy signals better. Therefore, minimizing the trajectory error of the noisy time series will no longer minimize at a faster pace the error of the real time series. That is why some prior knowledge about the scale in which the noise occurs is needed to select the right amount of harmonics.

\section{Discussion}

This paper presents a new way of using the evolutionary algorithms in the context of network reconstruction. Simulated annealing is indeed capable of improving the existing network reconstruction methods. The contribution of this paper is therefore primarily methodological since we propose a new reconstruction method. Additionally, we emphasize that the logic of evolutionary algorithms - in our case simulated annealing - can be fruitful for enhancing the existing or designing new network reconstruction methods, tangential to the problems in physics and other sciences.

On the other hand, limitations coming from different dynamical regimes seem to pose a considerable problem that requires additional research. As expected, for enough variability of fields in the dynamics the method performs better, as clear from all realizations. This opens the question of how to establish whether a given good reconstruction is also the best theoretically obtainable reconstruction under any method. In other words, how can we know if our method was able to extract all the information contained in the time series and use it for reconstruction. This amounts to computing the information content of time series, and examining its relation with the final precision. Our investigation of the method performance in more realistic scenarios seems to confirm such relation, which presents an interesting line of further work.

We also found that different time series attributes, such as measurement frequency or number of points, interplay in yielding the final precision, as they contribute to variation in the phase space coverage and derivative estimates. Of course, further adjustments of our method, such as temperature reduction scheme, can render it more applicable for a given specific scenario. Additionally, we showed that the method can be applied when noise is added to the time series. However, a more detail characterization of the effect of noise is a pending task.

The application of the method to bigger systems is still possible, however since the computation time grows with the system size, some optimization of the computing algorithm has to be made to go to bigger networks. Nevertheless, the application to realistic data sets requires the knowledge of the interaction functions $f$ and $h$. This has just been recently generalized in [16] where the specific knowledge of the functions details are not needed. Still, some a priori knowledge of the structure of the system is needed. So a method which uses small data sets and has as minimal assumptions as possible is an open problem. 
Evolutionary optimization of network reconstruction from derivative-variable correlations14

\section{Acknowledgment}

This work was founded by the EU via H2020 Marie SklodowskaCurie project COSMOS, grant no. 642563. $R G A$ acknowledges funding from the Volkswagen foundation, the Spanish Ministry of Economy and Competitiveness (Grant FIS2014-54177-R) and the CERCA Programme of the Generalitat de Catalunya. Z L acknowledges funding from the Slovenian Research Agency via program Complex Networks P1-0383 and project J58236.

\section{References}

[1] R. Albert and A.-L. Barabasi, "Statistical mechanics of complex networks," Rev. Mod. Phys., vol. 74, 2002.

[2] S. H. Strogatz, "Exploring complex networks," Nature, vol. 410, no. 6825, pp. 268-276, 2001.

[3] M. Newman, Networks, An Introduction. Oxford UP., 2007.

[4] R. Guimerà and M. Sales-Pardo, "Missing and spurious interactions and the reconstruction of complex networks," PNAS, vol. 106, no. 52, pp. 22073-22078, 2011.

[5] S. Hempel, A. Koseska, J. Kurths, and Z. Nikoloski, "Inner composition alignment for inferring directed networks from short time series," Phys. Rev. Lett., vol. 107, no. 5, p. 054101, 2011.

[6] E. S. Ching, P.-Y. Lai, and C. Leung, "Reconstructing weighted networks from dynamics," Phys. Rev. E, vol. 91, no. 3, p. 030801(R), 2015.

[7] X. Han, Z. Shen, W.-X. Wang, and Z. Di, "Robust reconstruction of complex networks from sparse data," Phys. Rev. Lett., vol. 114, no. 2, p. 028701, 2015.

[8] R.-Q. Su, W.-X. Wang, and Y.-C. Lai, "Detecting hidden nodes in complex networks from time series," Phys. Rev. E, vol. 85, no. 6, p. 065201, 2012.

[9] S. G. Shandilya and M. Timme, "Inferring network topology from complex dynamics," New J. Phys., vol. 13, no. 1, p. 013004, 2011.

[10] Y. Kuramoto, "Self-entrainment of a population of coupled non-linear oscillators," in International symposium on mathematical problems in theoretical physics, pp. 420-422, Springer, 1975.

[11] A. Arenas, A. Díaz-Guilera, J. Kurths, Y. Moreno, and C. Zhou, "Synchronization in complex networks," Physics reports, vol. 469, no. 3, pp. 93-153, 2008.

[12] M. Timme, "Revealing network connectivity from response dynamics," Phys. Rev. Lett., vol. 98, no. 22, p. 224101, 2007.

[13] Z. Levnajić and A. Pikovsky, "Network reconstruction from random phase resetting," Phys. Rev. Lett., vol. 107, no. 3, p. 034101, 2011.

[14] L. Prignamo and A. Díaz Aguilera, "Extracting topological features from dynamical measures in networks of kuramoto oscillators," Phys. Rev. E, vol. 85, p. 036112, 2012.

[15] Z. Levnajić and A. Pikovsky, "Untangling complex dynamical systems via derivative-variable correlations," Sci. Rep., vol. 4, p. 5030, 2014.

[16] A. Pikovsky, "Reconstruction of chaotic neural network from observed firing rates," Phys. Rev. E, vol. 93, p. 062313, 2016.

[17] G. Mei, X. Wu, G. Chen, and J.-A. Lu, "Identifying structures of continuously-varying weighted networks," Sci. Rep., vol. 6, p. 26649, 2016.

[18] W.-X. Wang, R. Yang, Y.-C. Lai, V. Kovanis, and M. A. F. Harrison, "Time-series-based prediction of complex oscillator networks via compressive sensing," EPL, vol. 94, no. 4, p. 48006, 2011.

[19] M. Ipsen and A. S. Mikhailov, "Evolutionary reconstruction of networks," Phys. Rev. E, vol. 66, p. 046109, 2002.

[20] N. Metropolis, A. W. Rosenbluth, M. N. Rosenbluth, A. H. Teller, and E. Teller, "Equation of state calculations by fast computing machines," J. Chem. Phys., vol. 21, no. 6, pp. 1087-1092, 1953. 
[21] S. V. Isakov, I. N. Zintchenko, T. F. Rønnow, and M. Troyer, "Optimized simulated annealing for ising spin glasses," Comput. Phys. Commun., vol. 192, pp. 265-271, 2015.

[22] M. Talukdar, O. Torsaeter, M. Ioannidis, and J. Howard, "Stochastic reconstruction, 3d characterization and network modeling of chalk," J. Pet. Sci. Eng., vol. 35, no. 1, pp. 1-21, 2002.

[23] L. Ingber et al., "Adaptive simulated annealing (asa): Lessons learned," Control and cybernetics, vol. 25, pp. 33-54, 1996.

[24] D. Hansel and H. Sompolinsky, "Solvable model of spatiotemporal chaos," Phys. Rev. Let., vol. 71, no. 17 , pp. 2710-2714, 1993. 